\title{
Secagem convectiva de cálice de hibisco: cinética e taxa de secagem
}

\author{
Convective drying of Roselle calyx: kinetics and drying rate \\ Secado convectivo de cálice de Jamaica: cinética y tasa de secado
}

Recebido: 20/01/2021 | Revisado: 27/01/2021 | Aceito: 31/01/2021 | Publicado: 07/02/2021

Mateus Vinicius de Paiva

ORCID: https://orcid.org/0000-0002-2919-510X Universidade Tecnológica Federal do Paraná, Brasil E-mail: mateusviniciuspaiva@outlook.com

Marcelo Zuchi Sanches

ORCID: https://orcid.org/0000-0003-0884-8201 Universidade Tecnológica Federal do Paraná, Brasil E-mail: marcelozsanches@hotmail.com

Leonardo Galice Chies

ORCID: https://orcid.org/0000-0002-9107-1802 Universidade Tecnológica Federal do Paraná, Brasil E-mail: leonardo.g.chies@hotmail.com

Admilson Lopes Vieira

ORCID: https://orcid.org/0000-0003-2979-4185 Universidade Tecnológica Federal do Paraná, Brasil

E-mail: lopesvieira@utfpr.edu.br

Joel Fernando Nicoleti

ORCID: https://orcid.org/0000-0002-3681-7253 Universidade Tecnológica Federal do Paraná, Brasil E-mail: nicoleti@utfpr.edu.br

Lyssa Setsuko Sakanaka

ORCID: https://orcid.org/0000-0002-3480-6593 Universidade Tecnológica Federal do Paraná, Brasil E-mail: lyssa@utfpr.edu.br

\begin{abstract}
Resumo
O cálice de hibisco é rico em ácido málico, antocianinas, ácido ascórbico, cálcio e ferro, o que o torna um ingrediente interessante, tanto seco como na forma de extrato em pó, de alimentos funcionais que auxiliam na promoção da saúde, bem-estar físico e mental. A secagem é uma operação unitária que agrega praticidade ao consumo de diversos alimentos, tendo ainda a vantagem de concentrar os seus compostos ativos, devido a remoção de umidade do material, estendendo sua vida útil, sem a necessidade de refrigeração, pela redução da atividade de água. O estudo da cinética de secagem traz informações importantes para o projeto e desenho de secadores, assim como, para a modelagem e simulação dos processos. Neste sentido, o objetivo do trabalho foi avaliar a cinética de secagem convectiva de cálices de hibisco utilizando uma estufa de secagem com convecção forçada de ar. Os cálices de hibisco foram dispostos em bandejas e submetidos à secagem nas temperaturas de 50,60 e $70{ }^{\circ} \mathrm{C}$ até peso constante. As amostras foram caracterizadas quanto à umidade e atividade de água. Cinco modelos matemáticos foram ajustados às curvas de secagem aplicando-se e o programa Microsoft Excel. O modelo de Page foi o que melhor descreveu o comportamento de secagem dos cálices de hibisco e dados estatísticos. Somente a taxa de secagem decrescente foi observada, com alta taxa no início da secagem, sendo gradualmente reduzida até o final do processo.
\end{abstract}

Palavras-chave: Hibiscus sabdariffa; Modelagem matemática; Umidade de equilíbrio.

\begin{abstract}
Roselle calyx is rich in malic acid, anthocyanins, ascorbic acid, calcium and iron, which make it an interesting ingredient, dried or as an extract powder, to functional foods that helps promote health, physical and mental wellbeing. Drying is a unit operation that adds practicality to the consumption of a great variety of foods, also having the advantage of concentrating the active compounds due to the removal of moisture from the material, extending its shelf life, without the need of refrigeration, by the reduction of water activity. The study of drying kinetics provides important information for the project and design of dryers, as well as for the processes modeling and simulation. Therefore, the objective of this work was to evaluate the kinetics of convective drying of Roselle calyces using a convective forced air oven. Roselle calyces were placed in trays and dried at temperatures of 50,60 and $70{ }^{\circ} \mathrm{C}$ until constant weight. Samples humidity and water activity were determined. Five models were used to fit drying curves using Microsoft Excel program. Page's model showed to be the best to describe the drying kinetics of Roselle calyces and statistics data. Only the decreasing drying rate was observed, with a high rate at the beginning of the drying, being gradually reduced until the end of the process.
\end{abstract}


Keywords: Hibiscus sabdariffa; Mathematical model; Equilibrium humidity.

\section{Resumen}

El cálice de Jamaica es rico en ácido málico, antocianinas, ácido ascórbico, calcio y hierro, lo que lo hace un ingrediente atractivo, sea seco o como extracto em polvo, a ser agregado a alimentos que promuevan la salud, el bienestar físico y mental. El secado es una operación unitaria que agrega practicidad al consumo de diferentes alimentos, teniendo además la ventaja de concentrar los compuestos activos debido a la remoción de humedad del material, extendiendo su vida útil, sin necesidad de refrigeración, por la disminucíon de su actividad del agua. El estudio de la cinética del secado permite obtener información importante para el proyecto y diseño de secadores, así como para la modelación y simulación del proceso. Así, el objetivo del trabajo fue evaluar la cinética del secado convectivo de cálices de Jamaica empleando un horno de secado con convección forzada del aire. Los cálices de Jamaica fueron colocadas sobre bandejas y secos a 50, 60 y $70{ }^{\circ} \mathrm{C}$ hasta peso constante. Las muestras fueron sometidas al análises de humedad y actividad del agua. Cinco modelos fueron empleados para el ajuste de las curvas del secado mediante el programa Microsoft Excel. El modelo cinético de Page describió mejor el comportamiento del secado de los cálices de Jamaica, así como resultados estadísticos. Solo se observó la tasa del secado decreciente, con una tasa alta al início del secado, reduciéndose gradualmente hasta el final del proceso.

Palabras clave: Hibiscus sabdariffa; Modelaje matemática; Humedad del equilíbrio.

\section{Introdução}

A planta do hibisco (Hibiscus sabdariffa) é popularmente conhecida como Vinagreira. Originária da África, foi rapidamente difundida na região nordeste do Brasil (Embrapa, 2017). Suas folhas são empregadas no preparo de condimentos, saladas cruas ou refogadas, as flores são usadas como matéria prima de chás, e dos cálices é comum o preparo também de chás, geleias e sucos (Brasil, 2010).

O cálice de hibisco é rico em compostos fitoquímicos com propriedades antioxidantes (Ochoa-Velasco et al., 2017). Rodrigues et al. (2020), ao estudarem a capacidade antioxidante do cálice da vinagreira, obtiveram teores de fenólicos totais no extrato aquoso, etanólico e acetônico em torno de 79,22; 83,63 e 80,02 mg equivalente de ácido gálico (EAG)/100g de amostra, respectivamente, e os três extratos apresentaram elevada capacidade de sequestrar radicais ABTS apresentando valores entre 34,925 mM de Trolox/g a 132,175 mM de Trolox/g, após 2 e 30 minutos de extração, respectivamente, sendo que esses valores se apresentaram maiores do que as obtidas para polpas como acerola, bacuri, goiaba e cajá, segundo os autores. Adicionalmente, Jung et al. (2013), avaliando a capacidade antimicrobiana do extrato de hibisco concluíram, em seu trabalho, um grande potencial de inibição da bactéria Escherichia coli.

A secagem do cálice de hibisco é uma alternativa que agrega praticidade no preparo do chá, além de, ao final da secagem, obter um produto com características nutricionais superior ao cálice in natura, pois com a remoção de água durante secagem, os compostos fenólicos, e, principalmente as antocianinas, são concentradas.

Daniel et al. (2013) empregaram secador de leito vibro fluidizado na secagem de cálices de hibisco e concluíram que os compostos fenólicos, antocianinas e atividade antioxidante dos cálices foram preservados. Nguyen e Chuyen (2020) secaram cálices de hibisco em secador convectivo de bandeja nas temperaturas de 60, 80, 100 e $120{ }^{\circ} \mathrm{C}$ até umidade final de $8 \%$; e obtiveram como resultado que o extrato de hibisco seco a $80 \circ \mathrm{C}$ foi o que apresentou maior atividade antioxidante (IC50 de $2,0 \mathrm{mg} / \mathrm{mL})$, e compostos fenólicos $(25 \mathrm{mg}$ de EAG/g), em relação às demais temperaturas. Também obtiveram bons resultados na análise sensorial, sendo que as amostras secas em temperaturas mais baixas $\left(60\right.$ e $\left.80^{\circ} \mathrm{C}\right)$ tiveram avaliações mais altas para os atributos de aroma, cor e gosto residual, enquanto as amostras desidratadas em temperaturas de secagem mais altas $\left(100\right.$ e $\left.120^{\circ} \mathrm{C}\right)$ resultaram em notas mais elevadas para o atributo sabor.

Além da qualidade nutricional e sensorial dos alimentos secos, é importante descrever o processo matematicamente, de forma que as equações obtidas possam ser utilizadas no próprio estudo da cinética de secagem para obtenção das taxas de secagem, ou mesmo em projetos de secadores, permitindo a compreensão do comportamento do material durante esse processo. As taxas de secagem podem ser crescentes, decrescentes ou constantes, dependendo da composição química do 
produto e/ou geometria do material. O período de taxa crescente é caracterizado pelo aumento da temperatura do produto até a temperatura de bulbo úmido do ar. A taxa constante baseia-se na teoria em que a velocidade de transferência de massa no interior do sólido poroso é igual à velocidade de evaporação da água na superfície do mesmo. Este fato considera somente o mecanismo de transferência de calor para o cálculo da taxa de secagem, assumindo que o calor que chega à superfície do sólido é removido pela evaporação da umidade (calor latente de vaporização).

Para predizer o comportamento no período de taxa de secagem decrescente empregam-se o modelo teórico, baseado na segunda lei de Fick, o modelo semi-teórico, que estabelece que a taxa de secagem é proporcional ao teor de água livre, e os modelos empíricos. Na secagem de chuchu (Ruiz-Lopes et al., 2012), batatas (Bakal et al., 2012), maçãs (Doymaz, 2010) e cálice de hibisco (Tajudin et al., 2019) os pesquisadores encontraram os valores da difusividade efetiva empregando o modelo teórico. Dentre os trabalhos que empregam os modelos empíricos e semi-empíricos sobre a cinética de secagem de espécies vegetais, pode-se citar Purkayastha et al. (2013) para tomates, Tunde-Akintunde e Ogunlakin (2013) para abóboras, Diamante et al. (2010) para damascos e kiwi.

Tajudin et al. (2019) compararam a cinética de secagem dos cálices de vinagreira em dois sistemas, um empregando bomba de calor e outro utilizando secagem solar, obtendo como resultado dois períodos de taxa de secagem - crescente e decrescente - não sendo observada taxa constante. O teor de umidade crítica, umidade onde se observa o início da taxa decrescente, ocorreu devido ao colapso da camada cerosa do cálice de hibisco, segundo os autores.

Muitos trabalhos que levam em consideração a qualidade das partes que constituem a planta de hibisco durante a secagem podem ser encontrados na literatura (Nguyen e Chuyen, 2020; Djaeni et al., 2018; Mar et al., 2020), porém há pouca informação sobre a cinética e o comportamento do material durante a secagem convectiva do cálice de hibisco. Desta forma, o objetivo deste trabalho foi determinar e caracterizar a cinética e as taxas de secagem de cálices de hibisco empregando um secador convectivo de bandejas em função da temperatura do ar de secagem.

\section{Material e Métodos}

Os cálices de hibisco, cultivados na região norte do Paraná, foram adquiridos em feiras livres na cidade de Londrina, PR, em outono de 2019. Os cálices foram cortados e separados das partes da planta e sementes, submetidos à higienização com água e enxaguados. Em seguida, foram centrifugados a 2.800 rotações por minuto (rpm) por 3 a 5 min, para remoção do excesso de água. Pesou-se uniformemente 100 gramas de cálices de hibisco em três bandejas (triplicata) as quais foram levadas à estufa de secagem (Marca Nova Ética) com convecção forçada de ar e após a mesma entrar em equilíbrio térmico nas temperaturas programadas, ou seja, 50, 60 e $70^{\circ} \mathrm{C}$. Em intervalo de 20 minutos, as bandejas eram pesadas e sua posição na estufa alternada para promover maior uniformização, caso houvesse algum gradiente de temperatura no interior da estufa. A secagem prosseguiu até o peso constante das bandejas, como um indicativo do final da secagem. Após esta etapa, as amostras secas foram armazenadas em potes herméticos de vidro para posteriores análises.

O conteúdo de umidade inicial foi obtido pelo método gravimétrico em estufa a $105^{\circ} \mathrm{C}$ por 24 horas (AOAC, 2006). A atividade de água foi medida pelo equipamento AquaLab Series 4TE. Os ensaios foram realizados em triplicata.

Os resultados de cinética de secagem foram avaliados a partir de cinco modelos matemáticos, nos quais os dois primeiros são semi-teóricos e os demais empíricos: Newton (Equação 1), Page (Equação 2), Henderson e Pabis (Equação 3), logarítmico (Equação 4) e dois termos (Equação 5).

$$
\begin{aligned}
& \text { Xad }=\exp (-a \cdot t) \\
& \text { Xad }=\exp \left(-a \cdot t^{b}\right)
\end{aligned}
$$




$$
\begin{aligned}
\mathrm{Xad} & =\mathrm{b} \cdot \exp (-\mathrm{a} \cdot \mathrm{t}) \\
\mathrm{Xad} & =\mathrm{b} \cdot \exp (-\mathrm{a} \cdot \mathrm{t})+\mathrm{c} \\
\mathrm{Xad} & =\mathrm{a} \cdot \exp (-\mathrm{b} \cdot \mathrm{t})+\mathrm{c} \cdot \exp (-\mathrm{d} \cdot \mathrm{t})
\end{aligned}
$$

Onde Xad é o conteúdo de umidade adimensional em base seca (b.s.) obtida de acordo com a razão [(X(t)-Xeq)/(XoXeq)], sendo Xo o conteúdo de umidade inicial (b.s) e Xeq o conteúdo de umidade de equilíbrio (b.s.); t o tempo de secagem (s) e "a, b, c, d" são as constantes dos modelos.

A estimativa dos parâmetros dos modelos foi realizada pelo método dos mínimos quadrados utilizando o programa Microsoft Excel (for Windows) e a função solver. A qualidade estatística da regressão não-linear foi avaliada pela função objetivo (S) e o desvio-padrão da estimativa (SE), de acordo com as equações (6) e (7). O coeficiente de determinação $\left(\mathrm{R}^{2}\right)$ também foi um parâmetro de avaliação.

$$
\begin{array}{ll}
S=\sum_{i=1}^{N}(Y-Y o)^{2} & \text { (Equação 6) } \\
S E=\sqrt{\frac{\sum_{i=1}^{N}(Y-Y o)^{2}}{N}} & \text { (Equação 7) }
\end{array}
$$

Onde Y representa o valor experimental, Yo o valor estimado pelo modelo, e $\mathrm{N}$ o número de observações experimentais.

\section{Resultados e Discussão}

O conteúdo de umidade inicial do cálice de hibisco empregado nos ensaios de secagem foi em média de 8,53 $\pm 0,86$ (b.s.). A Tabela 1 traz o detalhamento do teor de umidade inicial (Xo) e da umidade de equilíbrio (Xeq) ao final da secagem para as três condições de temperatura de secagem avaliadas. A Xeq se manteve abaixo de 0,2 (b.s), para todas as temperaturas de secagem, e a atividade de água dos cálices de hibisco estão abaixo de 0,60 (Tabela 1), considerado valor limite para garantia da estabilidade microbiológica de alimentos (Fennema, 2000). Assim sendo, pode-se afirmar que todas as temperaturas de secagem estudadas permitem a obtenção de um alimento seguro para consumo, desde que armazenado em embalagem apropriada. Em relação à vida útil dos cálices de hibisco secos, há a necessidade de ensaios microbiológicos e físico-químicos para estimativa do tempo apropriado para o consumo, em um ambiente com temperatura e umidade relativa controladas. 
Tabela 1. Teores de umidade inicial (Xo) e final (Xeq) e atividade de água (aw) para cada temperatura do ar de secagem.

\begin{tabular}{|c|c|c|c|}
\hline $\mathbf{T}\left({ }^{\circ} \mathrm{C}\right)$ & Xo (kg ag/kg ms) & Xeq (kg ag/kg ms) & aw \\
\hline 70 & $7,74 \pm 0,24$ & $0,180 \pm 0,018$ & $0,47 \pm 0,05$ \\
\hline 60 & $8,40 \pm 0,30$ & $0,088 \pm 0,016$ & $0,31 \pm 0,02$ \\
\hline 50 & $9,45 \pm 0,07$ & $0,140 \pm 0,065$ & $0,32 \pm 0,03$ \\
\hline
\end{tabular}

Fonte: Autores.

Os modelos cinéticos de secagem avaliados e seus respectivos parâmetros obtidos a partir do ajuste aos dados experimentais são apresentados na Tabela 2, em função das condições operacionais de temperatura de secagem. Os resultados estatísticos - coeficiente de determinação $\left(\mathrm{R}^{2}\right)$, função objetivo (S) e desvio padrão da estimativo (SE) - também estão apresentados.

Tabela 2. Valores dos parâmetros dos modelos cinéticos de secagem e resultados estatísticos de acordo com a temperatura do ar de secagem.

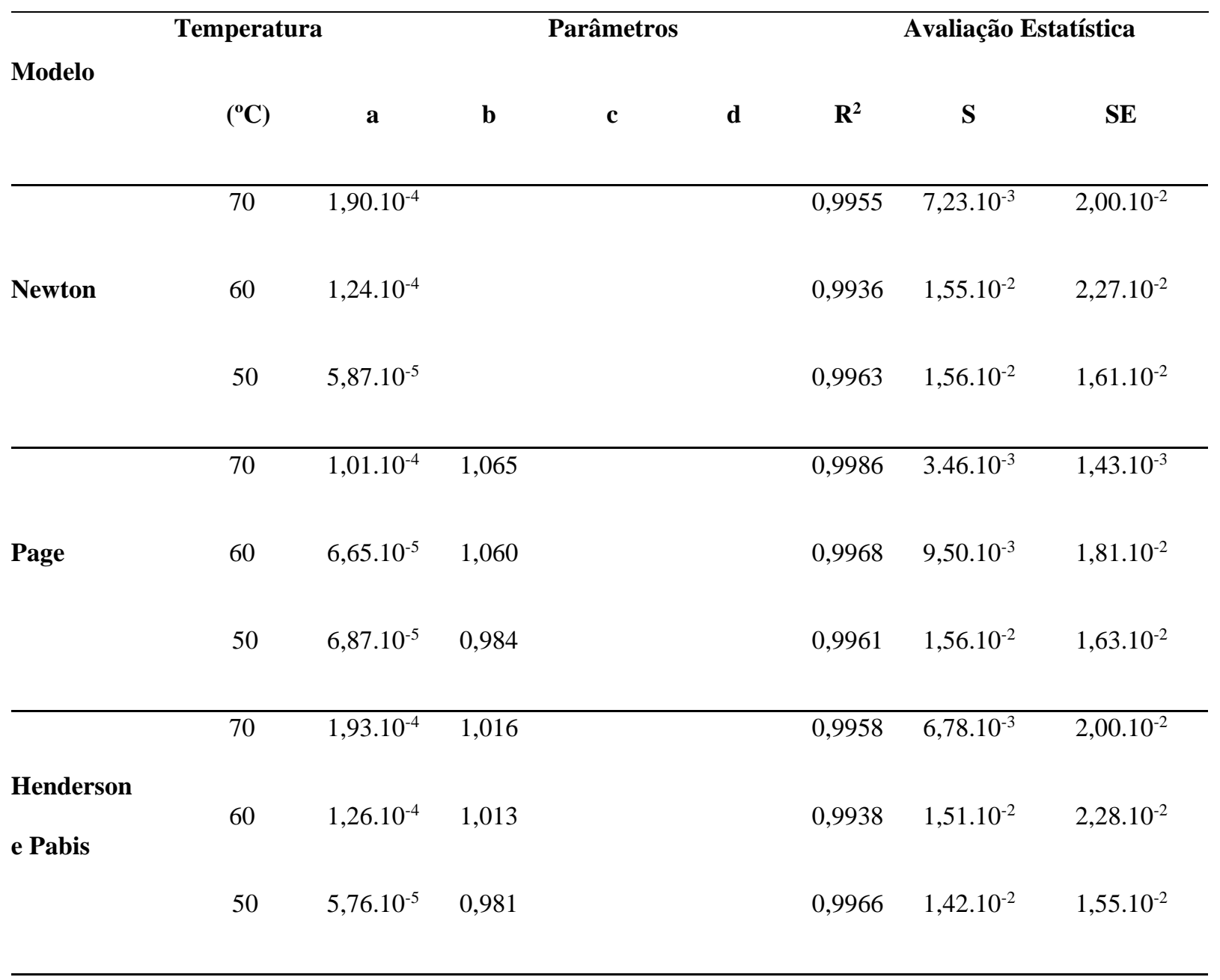


Research, Society and Development, v. 10, n. 2, e12910212224, 2021

(CC BY 4.0) | ISSN 2525-3409 | DOI: http://dx.doi.org/10.33448/rsd-v10i2.12224

70

$1,93.10^{-4} \quad 1,016$

0

0,9958

$6,78.10^{-3}$

$2,06.10^{-2}$

Logarítmico

60

$1,26.10^{-4} \quad 1,013$

0

$0,9938 \quad 1,51 \cdot 10^{-2}$

$2,32.10^{-2}$

50

$5,76.10^{-5} \quad 0,981$

0

$0,9966 \quad 1,42.10^{-2}$

$1,56.10^{-2}$

\begin{tabular}{lcccccccc}
\hline & 70 & 0,936 & $1,93.10^{-4}$ & $8,00.10^{-2}$ & $1,93.10^{-4}$ & 0,9958 & $6,78.10^{-3}$ & $2,13.10^{-2}$ \\
Dois termos & 60 & 0,913 & $1,26.10^{-4}$ & 0,100 & $1,26.10^{-4}$ & 0,9938 & $1,51.10^{-2}$ & $2,37.10^{-2}$ \\
& 50 & 0,941 & $4,76.10^{-5}$ & $4,08.10^{-2}$ & $5,76.10^{-5}$ & 0,9966 & $1,42.10^{-2}$ & $1,58.10^{-2}$ \\
& & & & & & & & \\
\hline
\end{tabular}

Fonte: Autores.

A partir dos resultados obtidos na Tabela 2 pode-se verificar que o melhor modelo aplicado à cinética de secagem do cálice de hibisco é o de Page, de acordo com os resultados estatísticos. Em temperaturas mais altas, o modelo tem uma maior representatividade ao resultado experimental observado pelos melhores $\mathrm{R}^{2}, \mathrm{~S}$ e SE. Neste sentido, optou-se em apresentar a curva do modelo de Page ajustado aos pontos experimentais, conforme Figura 1. Nesta figura também se observa uma melhor distribuição dos pontos experimentais à curva do modelo na temperatura mais alta.

Figura 1. Cinética de secagem dos cálices de hibisco em função da temperatura do ar de secagem.

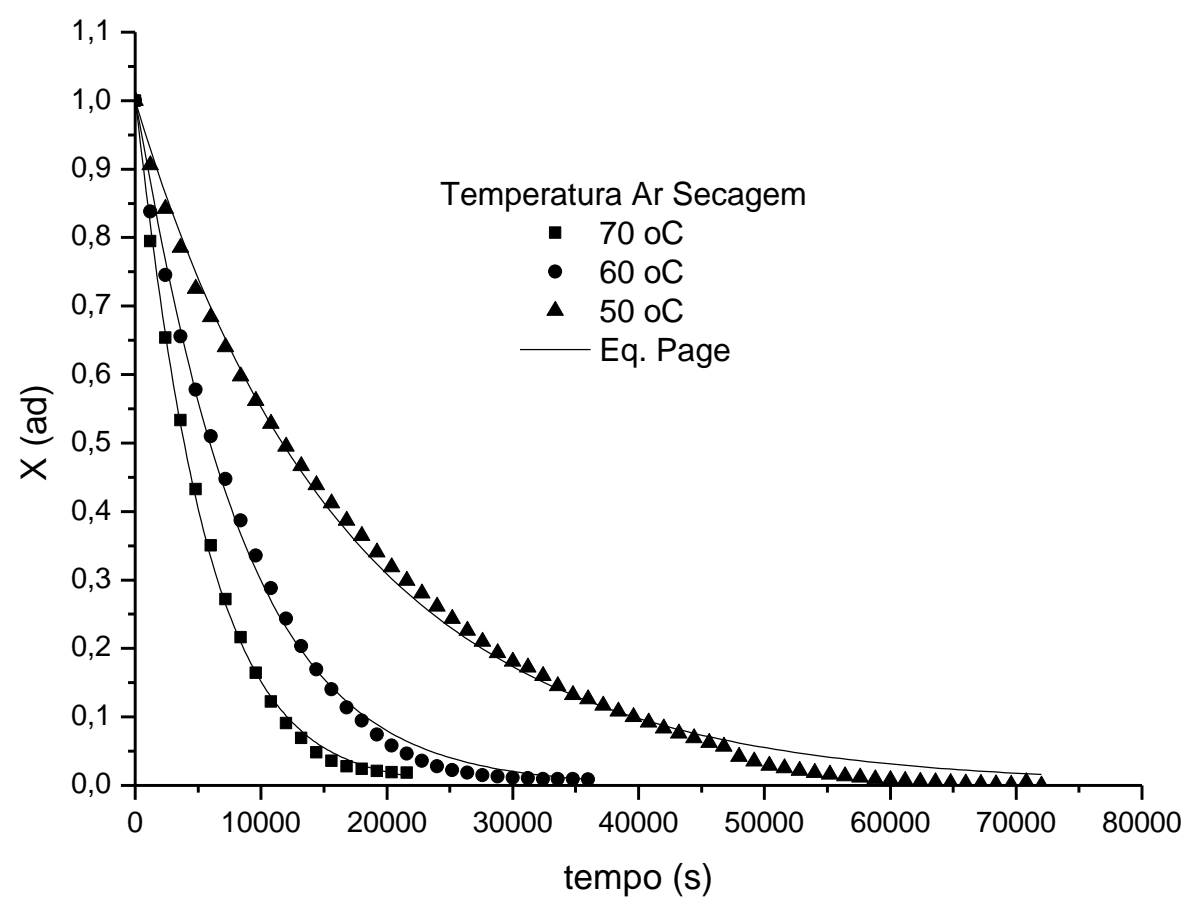

Fonte: Autores. 
É evidenciado pela Figura 1 um aumento no potencial de secagem com a elevação da temperatura do ar. Este efeito era esperado e diversos trabalhos convergem para o mesmo resultado (Demiray et al., 2017; Aral e Bese, 2016; Ostermeier et al., 2018). Fixando-se um teor de umidade adimensional (Xad) final para o cálice de hibisco em 0,1 observa-se um tempo necessário de permanência no secador, em média, de 12000, 18000 e 40000 segundos nas temperaturas de 70,60 e $50{ }^{\circ} \mathrm{C}$, respectivamente. Apesar das reações de degradação nutricional serem aumentadas com a elevação da temperatura, há de se considerar o tempo de exposição ao calor também. O material exposto a $70{ }^{\circ} \mathrm{C}$ pode ser que tenha menos prejuízo nutricional se comparada à temperatura de $50{ }^{\circ} \mathrm{C}$ devido ao menor tempo de contato com a fonte de calor. Esse fato também pode ser aplicado a outros parâmetros de qualidade como cor, textura, entre outros, necessitando de estudos adicionais focados na qualidade final do alimento seco.

A partir da derivada do teor de umidade (b.s.) em função do tempo de secagem é possível determinar as taxas de secagem. A Figura 2 apresenta as taxas de secagem em função das temperaturas do ar. Nenhuma fase crescente e constante de secagem foi observada, sendo este resultado encontrado também nas secagens de cebola (Demiray et al., 2017), fruta de espinheiro, Crataegus spp., (Aral e Bese, 2016) e marmelo (Izli e Polat, 2019). Por outro lado, Tajudin et al. (2019) encontraram um longo período de taxa crescente na secagem de cálices de hibisco.

A fase decrescente de secagem é caracterizada quando a taxa de migração de umidade do interior do material a ser seco é menor que a taxa de evaporação da superfície. Admite-se então que a superfície atingiu o teor de umidade de equilíbrio com o ar, enquanto o interior ainda contém significativas quantidades de água. A taxa decrescente é característica de transferência de massa em regime transiente e os ensaios experimentais e os modelos aplicados são bastante úteis na modelagem, simulação e projeto de secadores.

Figura 2. Taxas de secagem dos cálices de hibisco em função das temperaturas do ar de secagem.

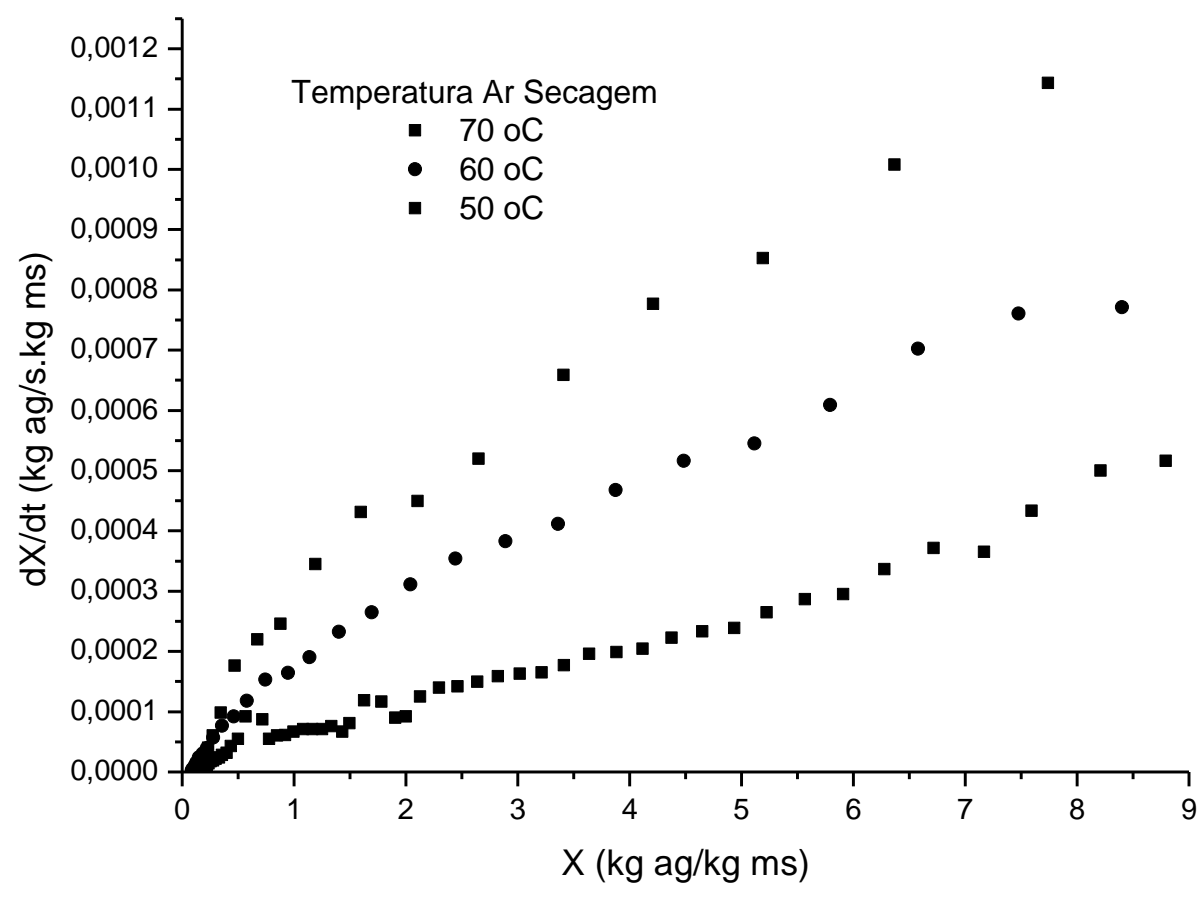

Fonte: Autores. 
No início da secagem, quando o alimento está com o teor de umidade elevado, as taxas de secagem são elevadas, sendo reduzidas conforme o teor de umidade é reduzido. Essa redução da taxa de secagem deve-se ao aumento da resistência de transferência de massa conforme a secagem avança, sendo que, no caso de gêneros alimentícios, é bastante prejudicial devido à elevação da temperatura da superfície do material, podendo chegar próximo à temperatura de bulbo seco do ar.

\section{Conclusões}

A cinética de secagem do cálice de hibisco foi fortemente influenciada pela temperatura do ar de secagem, conduzindo em um menor tempo de processo em temperaturas mais elevadas para o material atingir um conteúdo de umidade pré-estabelecido. O modelo de Page foi o mais apropriado para descrever o comportamento do produto durante a secagem, observado pelas análises estatísticas. As taxas de secagem seguiram somente a fase decrescente, sendo seus valores reduzidos com o avanço da secagem, indicando aumento na resistência de transferência de água do interior do material para sua superfície.

\section{Agradecimentos}

À UTFPR pela bolsa de extensão dos alunos MVP e LGC.

À CAPES, Fundação Araucária e ao CNPq pela infraestrutura.

\section{Referências}

AOAC. Official method of analysis (18th ed.). (2006). Washington, D.C: Association of Official Analytical Chemists.

Aral, S., \& Bese, A.V. (2016). Convective drying of hawthorn fruit (Crataegus spp.): effect of experimental parameters on drying kinetics, color, shrinkage and rehydration capacity. Food Chemistry, 210, 577-584.

Bakal, S. B., Sharma, G. P., Sonawane, S. P., \& Verma, R.C. (2012). Kinetics of potato drying using fluidized bed dryer. Journal of Food Science and Technology, 49(5), 608-613.

Brasil (2010). Ministério da Agricultura, Pecuária e Abastecimento. Hortaliças não-convencionais: (tradicionais) / Ministério da Agricultura, Pecuária e Abastecimento. Secretaria de Desenvolvimento Agropecuário e Cooperativismo. Brasília: MAPA/ACS. 52 p.

Demiray, E., Seker, A., \& Tulek, Y. (2017). Drying kinetics of onion (Allium cepa L.) slices with convective and microwave drying. Heat Mass Transfer, 53, $1817-1827$.

Diamante, L. M., Ihns,R., Savage,G. P., \& Vanhanen,L. (2010). A new mathematical model for thin layer drying of fruits. International Journal of Food Science \& Technology, 45(9), 1956-1962.

Djaeni, M., Kumoro, A., Sasongko, S. \& Utari, F. (2018). Drying Rate and Product Quality Evaluation of Roselle (Hibiscus sabdariffa L.) Calyces Extract Dried with Foaming Agent under Different Temperatures. International Journal of Food Science. 2018. 1-8. 10.1155/2018/9243549.

Doymaz, I. (2010). Effect of citric acid and blanching pre-treatments on drying and rehydration of Amasya red apples. Food and Bioproducts Processing, 88(2-3), 124- 132.

Embrapa hortaliças. (2017). Hortaliças não convencionais, hortaliças tradicionais. Folder Vinagreira. Brasília/Anápolis. Brasil.

Fennema, O. R. (2000). Química de los Alimentos. (2a ed.), Zaragoza: Acribia S.A. 1200p.

Izli, N. \& Polat, A. (2019). Freeze and convective drying of quince (Cydonia oblonga Miller.): Effects on drying kinetics and quality attributes. Heat and Mass Transfer. 55. 10.1007/s00231-018-2516-y.

Jung, E., Kim, Y., \& Joo, N. (2013). Physicochemical properties and antimicrobial activity of Roselle (Hibiscus sabdariffa L.). Journal of the Science of Food and Agriculture. 10.1002/jsfa.6256. Epub 2013 Jul 8. PMID: 23749748.

Leyva, D., et al (2013). Effect of drying conditions on the retention of phenolic compounds, anthocyanins and antioxidant activity of Roselle (Hibiscus sabdariffa L.) added to yogurt. International Journal of Food Science \& Technology. 48. 10.1111/ijfs.12215.

Mar, J., et al (2020). Bioactive compounds-rich powders: Influence of different carriers and drying techniques on the chemical stability of the Hibiscus acetosella extract. Powder Technology. 360. 383-391. 10.1016/j.powtec.2019.10.062.

Nguyen, Q. V., \& Hoang, C. (2020). Processing of Herbal Tea from Roselle (Hibiscus sabdariffa L.): Effects of Drying Temperature and Brewing Conditions on Total Soluble Solid, Phenolic Content, Antioxidant Capacity and Sensory Quality. Beverages. 6. 10.3390/beverages6010002. 
Research, Society and Development, v. 10, n. 2, e12910212224, 2021

(CC BY 4.0) | ISSN 2525-3409 | DOI: http://dx.doi.org/10.33448/rsd-v10i2.12224

Ochoa-Velasco, C. E., Salazar-Gonzales, C., Cid-Ortega, S., \& Guerrero-Beltran, J. A. (2017). Antioxidant characteristics of extracts of Hibiscus sabdariffa calyces encapsulated with mesquite gum. Journal of Food Science and Technology, 54 (7), 1747-1756.

Ostermeier, R., Giersemehl, P., Siemer, C., Topfl, S., \& Jager, H. (2018). Influence of pulsed electric field (PEF) pre-treatment on the convective drying kinetics of onions. Journal of Food Engineering, 237, 110-117.

Purkayastha, M., Amit Nath, A., Deka, B. C., \& Mahanta, C. L. (2013). Thin layer drying of tomato slices. Journal of Food Science and Technology, 50 (4), $642-653$.

Rodrigues, L. R., Sousa, M. M. D., Silva, J. N., Viana, L. T. M., Gomes, F. O., Sousa, C. R. N., Andrade, F. S., \& Lima, A. (2020). Vinagreira (Hibiscus sabdariffa L.): determinação do teor dos polifenóis totais e atividade antioxidante. Brazilian. Journal of Development. 6 (11), 89305-89312.

Ruiz-Lopez, I. I., Ruiz-Espinosa, H., Arellanes-Lozada, P., Barcenas-Pozos, M. E., \& Garcia-Alvarado, M. A. (2012). Analytical model for variable moisture diffusivity estimation and drying simulation of shrinkable food products. Journal of Food Engineering, 108 (3), 427-435.

Tajudin, N. H. A., Tasirin, S. M., Ang, W. L., Rosli, M. I., \& Lim, L. C. (2019). Comparison of drying kinetics and product quality from convective heat pump and solar drying of Roselle calyx. Food and Bioproducts Processing, 118, 40-49.

Tunde-akintunde, T. Y., \& Ogunlakin, G.O. (2013). Mathematical modeling of drying of pretreated and untreated pumpkin. Journal of Food Science and Technology, 50 (4), 705-713. 